\title{
ADMINISTRATION OF BIOFLAVONOIDES IMPROVES PLASMA LEVELS OF ADIPOCYTE HORMONES
}

\author{
M. Boncheva ${ }^{1}$ and T. Turnovska ${ }^{2}$ \\ ${ }^{1}$ Medical University - Varna, BG \\ ${ }^{2}$ Medical University - Plovdiv, BG
}

\begin{abstract}
Summary. Since time immemorial the fruits of aronia melanocarpa (rich of bioflavonoides) have been known for their medicinal properties. Present-day research of the pharmacological effects of aronia melanocarpa juice and fruits intake indicates that their high contents of anthocyanins is closely related to the health enhancing properties of this plant. This is a key fact which can be used in the prevention of most commonly spread, socially significant diseases, reducing for instance the total risk of cardio-vascular diseases. The great molecular variety anthocyanins possess and the role they play in cell metabolism, are still being investigated. This gives grounds to study the effects of Aronia melanocarpa on human cells, tissues, and organs. The aim of this study is to trace the effect of 150-200 ml aronia melanokarpa juice daily oral intake on the adipocyte hormones leptin (Lp), resistine (Rs) and adiponectin (Adn) blood levels in 10 patients with high body mass index (BMI, $\left.\mathrm{kg} / \mathrm{m}^{2}\right)$ and high waist circumference. We used ELISA methods for hormonal analyses. During the study-period of two months patients did not change anything in their lifestyle. In the study group, the levels of Rs, Lp and Adn changed significantly compared to their baseline levels (averages, $\mathrm{ng} / \mathrm{mL}$ ) $-6.93 \pm 0.137,18.40 \pm 1.021$ and $7.98 \pm 0.077$ vs. $5.06 \pm 0.011,15.23 \pm 0.906$ and $10.45 \pm 0.103$ at the end of the second month, respectively. Compared with the control group of 6 people, matched for BMI, not receiving aronia melanocarpa juice, these values were markedly different. Patients taking aronia melanokarpa juice report improvement in various conditions that have caused them discomfort before the research started: pain in the muscles and joints faded away and were replaced by a new feeling of strength, headache attacks disappeared, improvement in memory and sleep were reported, regular defecation, no signs of gastric discomfort, better vision, a quicker auditory reaction, motivation for having sex, good mood were also reported. Obese patients with insulin resistance have decreased serum adiponectin and increased serum resistin and leptin. The levels of these three hormones changed positively after chronic intake of aronia juice. We recommend a daily intake of aronia juice for the prevention of health.
\end{abstract}

Key words: aronia melanocarpa, resistine, leptin, adiponectin 


\section{INTRODUCTION}

W e present preliminary results in enhancing the secretion of certain hormones in fat cells after administration of aronia melanocarpa juice.

The homeland of Aronia bushes is North America. The fruits of this plant have been known for their medicinal properties since ancient times.

Today, we rediscover this fruit as a true, useful food, which has, so far, been only incidentally sought in states of disease coming close to the incurable ones. Current research points to the necessity of including this food in our daily diet as a grace to the healthy lifestyle [1].

The aronia fruit can be consumed as fresh fruit, cold pressed juice from fresh fruits, the so-called "fruit" wine, or marmalades. Due to the seasonal character of the crop, fruits are often offered dried or frozen.

Aronia is a genus in the family Rosaceae. It includes two basic types of deciduous shrubs: Aronia melanocarpa - black fruits, and Aronia arbutifolia - red fruits. The shrubs reach the height of $90-180 \mathrm{~cm}$. They blossom in April and the fruits ripen in end-August. The berries of Aronia melanocarpa are dark violet to black in color, and grow in clusters of 11-14 with a diameter of $6 \mathrm{~mm}$ [6].

Bulgaria is a country with extremely favourable climatic conditions and a lot of sites offering suitable soil and precipitation levels for the planting and growth of Aronia melanocarpa. Specifically, the sun-shine hours in our latitude allow for ripening of fruits with the highest proven contents of biologically active useful substances in comparison to other European countries. The beginning of cultivated growth of aronia in this country dates back to 1995. There are EC-certified biopruducers of aronia fruits and products (juice, fruit wine, marmalade) in Bulgaria [4].

A number of studies have been conducted on aronia fruits over the past 15 years. On one hand, the chemical composition of the fruits and the juice they contain has been investigated [7]. On the other hand, the specific effects of this juice on patterns of different diseases have been established. The patterns were provided by mice or cellular cultures of human cells taken from various impaired organs [1].

The cold pressed juice of fresh, not frozen fruits contains bioflavonoids - essential natural polyphenol compounds like anthocyanins, catechin, quercetin. These natural antioxidants are essential to human organism to cope with oxidative stress, which is considered the base of all diseases. There is no other plant with such record contents of polyphenol substances (P-active, vitamin P). Aronia contains five times the amounts of these substances as compared to grapes, grapefruit, and the beetroot. The presence of minerals and microelements such as iodine, potassium, calcium, phosphorus, magnesium, and iron is noteworthy, with the highest concentrations being these of iodine and manganese. The fruits have rich complex of vitamins: A, B1, B2, B3, B5, B6, B9, C, E, K, P and traces of B17. Carbohydrates comprise approximately $15 \%$. Small quantities of fructose required to satisfy daily needs; sorbitol and parasorbid, which attribute some weak to moderate laxative effects to the drink, are also present. Adequate concentrations of the ESSENTIAL 
amino acids - arginine, lysine, histidine, threonine, as well as of the non-essential amino acids - tyrosine, cysteine, $\alpha$-alanine, aspartic acid, serine, glutamine, are also part of the content $[7,8]$.

Polyphenols, in particular, anthocyanins and procyanidins show the highest concentration of all compounds. These components account for the anti-oxidative activity of the plant. Laboratory studies of the anti-oxidative effects of aronia juice/ the so-called ORAC - oxygen radical absorbance capacity/ show record values 160.62 Trolox Equivalents. In comparison to values shown by other 277 similar "red" plants, these values are multiple times higher [1].

The polyphenol substances found in aronia juice have been identified by the use of contemporary analytical technologies. Polymer proanthocyanins (epicatechin) have been found. High concentration of phenol acids - chlorogenic and neochlorogenic, as well as of quercetin has been established [8].

Scientific studies aim to prove the role of bioflavonoids, especifically of anthocyanins, in neutralizing free radicals stored in the body. Bioflavonoids show a more powerful neutralizing effect than classic antioxidants, such as vitamins $C$ and $E$ and beta-carotene (Vit $\mathrm{C}$ and beta-carotene are also provided by aronia juice intake). It is widely known that damage caused by free radicals underlies the emergence of most present-day socially significant diseases. This is why a multifaceted healing effect has been manifested with models of diseases after the course of aronia juice intake [9].

Over the recent years favorable changes at the genetic level have been reported following courses of aronia juice intake. These were mostly experiments with animals [9].

Clinical investigations with people are a bit more limited, however, the results in humans are concurrent with these reported for animals and cellular culture patterns. It can then be concluded that the consumption of aronia juice does not have any toxic effects. Eventual over-dosage may have a laxative effect which is terminated through dosage reduction, and this depends on individual circumstance [2].

\begin{abstract}
AIM
The aim of this study is to trace the effect of $150-200 \mathrm{ml}$ aronia melanokarpa juice daily oral intake on the adipocyte hormones leptin(Lp), resistine (Rs) and adiponectin (Adn) blood levels in 10 patients with high body mass index (BMl, $\left.\mathrm{kg} / \mathrm{m}^{2}\right)$ and high waist circumference.
\end{abstract}

\title{
PATIENTS AND METHODS
}

The group consisted of 16 patients -8 males and 8 females (Table 1). Ten patients were monitored by laboratory measurements at baseline and after two months of intake of aronia melanocarpa juice in doses 170-200 ml daily. Six patients (control group) were monitored by laboratory measurements at baseline and after two 
months of no intake of aronia melanocarpa juice. The serum concentrations of the three hormones - leptin (Lp), resistine (Rs) and adiponectin (Adn) were measured. We used ELISA methods for hormonal analyses. We also analyzed some routine laboratory parameters of carbohydrate and lipid metabolism. All participants filled out a short questionnaire about their complaints and self-reported health before and after taking the aronia juice. During the study-period of two months patients did not change anything in their lifestyle.

Table 1. Baseline characteristics of study subjects in control and aronia juice intake groups

\begin{tabular}{|l|c|c|c|c|c|}
\hline $\begin{array}{l}\text { Patients } \\
\text { groups }\end{array}$ & $\begin{array}{c}\text { Number } \\
\text { of people }\end{array}$ & $\begin{array}{c}\text { Age (years, } \\
\text { mean values) }\end{array}$ & $\begin{array}{c}\text { Body mass index (BMI) } \\
\left.\text { (mean values, } \mathrm{kg} / \mathrm{m}^{2}\right)\end{array}$ & $\begin{array}{c}\text { Waist circumference } \\
\text { (mean values-cm) }\end{array}$ & $\begin{array}{c}\text { Intake of aronia juice } \\
(\mathbf{1 7 0 - 2 0 0} \mathrm{ml} \mathrm{daily)}\end{array}$ \\
\hline $\begin{array}{l}\text { Aronia juice } \\
\text { intake group }\end{array}$ & 10 & 47.1 & 28.99 & 111.02 & with \\
\hline males & 7 & 45.86 & 27.4 & 116.70 & with \\
\hline females & 3 & 50 & 29.4 & 97.73 & with \\
\hline Control group & 6 & 47.5 & 28.9 & 96.22 & without \\
\hline males & 1 & 42 & 27.7 & 118,3 & without \\
\hline females & 5 & 48.6 & 29.2 & 91.8 & without \\
\hline $\begin{array}{l}\text { Total (mean } \\
\text { value) }\end{array}$ & 16 & 47.25 & 28.945 & 105.47 & \\
\hline
\end{tabular}

\section{RESULTS}

In the study group, the levels of Rs, Lp and Adn changed significantly compared to their baseline levels (averages, $\mathrm{ng} / \mathrm{mL}$ ) $-6.93 \pm 0.137,18.40 \pm 1.021$ and $7.98 \pm 0.077$ vs. $5.06 \pm 0.011,15.23 \pm 0.906$ and $10.45 \pm 0.103$ at the end of the second month, respectively. Compared with the control group of 6 people, matched for $\mathrm{BMI}$, not receiving aronia melanocarpa juice, these values were markedly different (Table 2). There were no statistically significant changes in the measures of lipid metabolism in between the two groups. Significant improvement in carbohydrate metabolism is expressed by percent reduction of $\mathrm{HbA} 1 \mathrm{c}(\%)$ values in patients taking aronia juice. Patients taking aronia melanokarpa juice reported improvement in various conditions that have caused them discomfort before the research started: pain in the muscles and joints faded away and were replaced by a new feeling of strength, headache attacks disappeared, improvement in memory and sleep were reported, regular defecation, no signs of gastric discomfort, better vision, a quicker auditory reaction, motivation for having sex, good mood were also reported. In comparison to the group that did not take aronia melanocarpa juice, the individual perception of feeling healthy was significantly improved among the participants taking aronia. 
Table 2. Changes in blood concentrations of leptin (Lp), resistine (Rs) and adiponectin (Adn) in patients without changes in lifestyle and with aronia melanocarpa juice intake (mean values $\pm \mathrm{SD}$ )

\begin{tabular}{|l|c|c|}
\hline Laboratory parameters & Basal values $(\mathrm{ng} / \mathrm{mL})$ & Values after 60 days $(\mathrm{ng} / \mathrm{mL})$ \\
\hline Patients with intake of aronia juice $(\mathrm{n}=10)$ & $18.40 \pm 1.021$ & $15.23 \pm 0.906$ \\
\hline Leptin (Lp), & $6.93 \pm 0.137$ & $5.06 \pm 0.011$ \\
\hline Resistine (Rs) & $7.98 \pm 0.077$ & $10.45 \pm 0.103$ \\
\hline Adiponectin (Adn) & $6.43 \pm 0.12$ & $5.22 \pm 0.03$ \\
\hline Hb A1c (\%) & $17.26 \pm 1.51$ & $17.96 \pm 1.95$ \\
\hline Control patients without intake of aronia juice $(\mathrm{n}=6)$ & $6.98 \pm 0.211$ \\
\hline Leptin (Lp) & $7.02 \pm 0.099$ & $8.179 \pm 0.291$ \\
\hline Resistine (Rs) & $8.053 \pm 0.360$ & $6.53 \pm 0.82$ \\
\hline Adiponectin (And) & $6.68 \pm 0.38$ & \\
\hline Hb A1c & &
\end{tabular}

\section{DISCUSSION}

The main feature and one of the first clinical symptoms of metabolic syndrome is the increase of body weight, especially of adipose tissue [3]. This is commonly related to unhealthy diet and a sedentary lifestyle. When disruption in energy balance ensues, i.e. taking more energy than the body consumes, that results in increased circulation of non-esterified fatty acids. They accumulate as triglycerides as reserves in the cells of white adipose tissue. This increases the waist circumference. There are two types of white fat cells - small and large. Small cells change by hyperplasia and larger cells - by hypertrophy. When the fill capacity of the fat cells is exceeded, accumulation of circulating non-esterified fatty acids leads to impairment of insulin receptors in peripheral tissues and insulin resistance occurs. Cells of white adipose tissue have several functions: storing energy in the form of triglycerides; protecting other organs and tissues from ectopic fat accumulation; exhibiting lipotoxic effects [4]. Until 1990, only the first two of these functions had been established. Later it was found that the hormone leptin, which is secreted by the adipocytes of white adipose tissue, transfers information to the central nervous system and plays a role in the regulation of energy reserves [10].

Metabolic syndrome starts with the accumulation of visceral fat. Adipose tissue is an important endocrine organ that secretes numerous protein hormones, including leptin, adiponectin, and resistin. These hormones generally influence energy metabolism, which is of great interest to scientists as a key to understanding the pathogenesis of type 2 diabetes mellitus and obesity and ,therefore, their treatment. Their relative roles in modifying appetite, insulin resistance and atherosclerosis are 
the subjects of intense research, as they may be modifiable causes of morbidity in people with obesity $[2,4,10]$.

Leptin is exclusively produced in white adipocytes and released to systemic circulation. Plasma leptin concentrations increase in proportion to body fat mass and thus can be used as biomarker of adiposity. Circulating leptin enters the brain through the blood-brain barrier (BBB) and the blood-cerebrospinal-fluid barrier (CSF) barriers through receptor-mediated mechanisms. Leptin receptors are highly expressed in the neurons of the hypothalamus, especially the arcuate nucleus (ARC) [10].

Resistin expression was greater in white adipose tissue than in brown adipose tissue. It has been observed that circulating resistin levels are increased in obese humans. It is considered a pro-inflammatory molecule, which also plays an important role in the pathogenesis of diabetes and its complications. The release of resistin is often stimulated by the inflammatory process, IL-6, hyperglycemia and hormones such as growth hormone and gonadal hormones [2, 10].

Adiponectin was abundantly present in the plasma of healthy volunteers in the range from 1.9 to $17.0 \mathrm{mg} / \mathrm{ml}$. Plasma concentrations of adiponectin in obese subjects were significantly lower than those in non-obese subjects, although adiponectin is secreted only from adipose tissue. Adiponectin has been postulated to play an important role in the modulation of glucose and lipid metabolism in insulin-sensitive tissues in humans. Decreased circulating adiponectin levels have been demonstrated in genetic and diet-induced murine models of obesity, as well as in diet-induced forms of human obesity. Low adiponectin levels have also been strongly implicated in the development of insulin resistance $[2,10]$.

The patients studied were not taking foods rich in bioflavonoids before the study. The results of this study suggest that prolonged daily intake of aronia juice will favorably influence the parameters of lipid metabolism and immunity. Health effects of bioflavonoids have been described in many studies. They positively affect cell mRNA and gene expression in all cells and organs [5, 11]. Overweight patients should be advised, together with taking aronia juice on a regular daily basis, to change their dietary habits and modify their lifestyle. "More movement and less energy intake" would be a thoughtful recommendation.

\section{CONCLUSION}

Obese patients with insulin resistance have decreased serum adiponectin and increased serum resistin and leptin. The levels of these three hormones changed positively after chronic intake of aronia juice. We recommend a daily intake of aronia juice for the prevention of health.

Currently, there are no data in the literature about any unwanted and toxic effects of Aronia melanocarpa fruits, juice and extracts, so chronic consumption for years should be completely safe. 


\section{REFERENCES}

1. Boncheva, M. Curative effects of the fruits of Aronia melanocarpa. - General medicine, 14, 2012, № 3, 39-48.

2. B on ch e va, M, G. Georgiev et V. Shishkov. Intake of aronia melanocarpa juice improves medical tests and the feeling of health in patients with non-alcoholic fatty liver disease. - General medicine, 15, 2013, № 2, 21-30.

3. Boncheva, M, G. Tzolova et R. Dimova. Metabolic syndrome - The GP's knowledge for this problem in Bulgaria. - Social medicine, 4, 2006, 5-8.

4. Ge orgiev, G., M. Boncheva et M. Jordanova. Admission of juice aronia melanocarpa improves medical research and feeling for health in patients with metabolic syndrome. 22nd International Congress of Clinical Chemistry and Laboratory Medicine, 22-26 June, Istanbul, Turkey. Clinical Chemistry and Laboratory Medicine, abstract 0749, S922.

5. B o h ky ung, K. et al. Polyphenol-rich Aronia melanocarpa (chokeberry) extract regulates expression of cholesterol and lipid metabolism genes in Caco-2 cells. - FASEB J., 26, 2012, 251-262.

6. Comprehensive Reviews in Food Science and Food Safety, 11, 2012.

7. Agric, J. Chemical Compounds of aronia melanocarpa fruit. - Food Chem., 51, 2003, № 2, $142-149$.

8. Kulling, S. E. et al. Chokeberry (aronia melanocarpa) chemical compaunds. - Planta Med., 74, 2008, 1625-1634.

9. M a n a c h, C., G. Williamson, C. Morand, A. Scalbert et C. Rémésy. Bioavailability and bioefficacy of polyphenols in humans. I. Review of 97 bioavailability studies. - Am. J. Clin. Nutrition, 81, 2005, 230S-242S.

10. S t o fk ova, A. Leptin and adiponectin: from energy and metabolic dysbalance to inflammation and autoimmunity. - Endocr. Regul., 18, 2009, 157-168.

11. Solin a s, G. et M. Karin Molecular links between obesity and metabolic dysfunction. - FASEB J., 18, 2010, 2596-2611.

\section{Corresponding author:}

Margaritka Boncheva, MD, PhD

Department of Clinical Laboratory

Medical University

55 Marin Drinov st.

Bg - 9000 Varna

e-mail: mbon@mail.bg 